\title{
In vitro biomimetic HPLC and in vivo characterisation of GM6, an endogenous regulator peptide drug candidate for amyotrophic lateral sclerosis
}

\author{
Klara Valko* ${ }^{1,2}$, Mark Kindy ${ }^{3,4}$, James Evans ${ }^{5}$, Dorothy Ko ${ }^{6}$ \\ ${ }^{1}$ Bio-Mimetic Chromatography Ltd, Business \& Technology Centre, Stevenage, SG1 2DX United Kingdom \\ ${ }^{2}$ Department of Biological and Pharmaceutical Chemistry, UCL School of Pharmacy London WC1N 1AX United Kingdom \\ ${ }^{3}$ Department of Pharmaceutical Sciences, College of Pharmacy, University of South Florida, Tampa, FL, USA. \\ ${ }^{4}$ James A. Haley VAMC, Tampa, FL, USA. \\ ${ }^{5}$ PhenoVista, San Diego, CA, USA \\ ${ }^{6}$ Genervon Biopharmaceuticals LLC, Pasadena, CA, USA
}

*Corresponding Author e-mail: K.Valko@ucl.ac.uk and Klara Valko@bio-mimetic-chromatography.com; Tel.: +44 7521989558

Received: May 08, 2018; Revised: May 28, 2018; Published: June 16, 2018

\begin{abstract}
Amyotrophic lateral sclerosis (ALS) is an idiopathic, fatal neurodegenerative disease of the human motor system. Subunits of the 33-amino acid containing motorneurontrophic factor (MNTF) have been investigated and GM6 has been found as a potential peptide therapeutic for ALS. This linear peptide drug candidate has been characterized by HPLC based physicochemical and biomimetic measurements to estimate its in vivo distribution behavior and to estimate its cell penetration and brain to plasma concentration ratio. The free tissue concentration vs time profile has been estimated using the measured physicochemical and biomimetic properties of the intact GM6 molecules and its microsomal stability. The in vitro and in vivo measurements supported the estimated in vivo distribution behavior of GM6.
\end{abstract}

\section{Keywords}

motoneuron degeneration; peptide drug candidate; ADME of peptide drugs; biomimetic properties; PK profile; free tissue concentration vs time PK

\section{Introduction}

Amyotrophic lateral sclerosis (ALS) is an idiopathic, fatal neurodegenerative disease of the human motor system. It is the most common motoneuron disease in human affecting approximately 2 people in 100,000 in both the US and Europe and therefore it is considered as a rare orphan disease [1]. ALS manifests itself in the death of motoneurons which control the voluntary muscles. The cause of ALS is not known in 90 to $95 \%$ of cases while familial inherited causes have been identified in 5 to $10 \%$ of cases. There is no cure for ALS. The clinical onset of the disease usually starts at around age 60 and the survival time is around 2 to 4 years. It is very difficult to determine a specific date for the onset of the disease and the potentially long duration between the onset of pathological changes and the manifestation of the clinical disease. Patients can present with bulbar-onset disease (about $25 \%$ ) or limb-onset disease (about 
$70 \%)$ or with initial trunk or respiratory involvement (5\%). Atypical modes of presentation can include weight loss, which is an indicator of a poor prognosis, cramps, emotional lability and frontal lobe-type cognitive dysfunction.

The pathophysiological mechanisms underlying the development of ALS seem multifactorial with emerging evidence of a complex interaction between genetic and molecular pathways [2,3]. ALS is relentlessly progressive $-50 \%$ of patients die within 30 months of symptom onset and about $20 \%$ of patients survive between 5 - 10 years after symptom onset. Some ALS subtypes tend to lead to a better prognosis; for example, flail-limb variants ALS and progressive muscular atrophy. Both are predominantly lower motoneuron forms that are characterised by slower progression. Advances in the understanding of the glutamate neurotransmitter system and the discovery of causal genes linked to the development of familial ALS has stimulated research interest. The survival in ALS is now understood to be dependent on several factors, such as clinical presentation, the rate of disease progression, early presence of respiratory failure and the nutritional status of the patients.

The process by which motoneurons die remains unclear with several inter-related mechanisms suggested [1]. The neurodegeneration in ALS may result from any of the following: complex interactions of glutamate excitotoxicity, generation of free radicals, cytoplasmic protein aggregates, SOD1 enzymes, combined with mitochondrial dysfunction, and disruption of axonal transport processes through the accumulation of neurofilament intracellular aggregates.

Riluzole has been approved by the FDA for 20 years and inhibits the release of glutamic acid from cultured neurons, from brain slices, and from corticostriatal neurons in vivo. It increases the ALS patients' life by two to three months by blocking glutamatergic neurotransmission in the CNS.

There is an unmet medical need to lengthen the life expectancy, slow down the disease progression and ultimately cure ALS patients. Discovering a therapeutic agent that significantly increases the life expectancy of ALS patients or cures the disease would provide a significant breakthrough in this field.

\section{Potential disease mechanisms}

Protein misfolding and protein aggregation are known characteristics of various diseases, including major neurodegenerative disorders such as ALS, Alzheimer's and Parkinson's diseases. While the mechanistic link between protein aggregation and cell degeneration remains unclear, approaches to halt or reverse the aggregation process are a focus of current drug discovery approaches.

Insulin-like growth factor-I (IGF-I) receptors are present in the spinal cord and, as members of the neurotrophin receptor family, IGF-I receptors mediate signal transduction via the tyrosine kinase domain. IGF-I was found to prevent the loss of choline acetyltransferase activity in embryonic spinal cord cultures, as well as reducing the programmed cell death of motor neurons in vivo during normal development or following axotomy [4]. Over the past 30 years, glucose intolerance has been reported in a significant percentage of ALS patients. A reduction in glucose receptor space and insulin resistance has been postulated for a number of neuromuscular diseases [5]. The structurally related peptides, insulin and insulin-like growth factors (IGF-I and IGF-II), have neurotrophic properties and potentially could be of therapeutic value in human neurodegenerative disorders [6].

The nerve growth factor (NGF) was discovered by Levi-Montalcini and Cohen some 50 years ago [7] which supported the concept that secreted molecules produced by the target of a developing neuron are required for it to survive programmed cell death (PCD). This neurotrophic hypothesis provided the 
background for the identification of neurotrophic factors (NTFs). The motor neuron trophic factor (MNTF) is a 33-amino acid peptide. It was identified, isolated and characterized by using monoclonal antibodies against a $35 \mathrm{kD}$ rat peptide, isolated from 3-week-old rat muscle extract. MNTF was subsequently derived by the cloning of a recombinant MNTF1-F6 gene from a human retinoblastoma cDNA expression library (Clontech) and analyzed to determine its amino acid and cDNA sequences [8]. GM6 is a 6 amino acid analogue of the MNTF active site. GM6 is a cationic linear peptide drug consisting of 6 amino acids in their natural configurations (Phe-Ser-Arg-Tyr-Ala-Arg acetate salt) $[9,10]$. MNTF $33(1 \mu \mathrm{g} / \mathrm{ml})$ and MNTF 10 $(1 \mu \mathrm{g} / \mathrm{ml})$ induced differentiation of embryonic stem cells (ES) that resulted in the expression of the mature motor neuron transcription factors HB9, Chat and Islet 1/2. MNTF was found to have an affinity for the insulin receptor (IR) and the Insulin-like growth factor (IGF) of ES cells that resulted in the autophosphorylation of Tyr972 and Tyr 1162/1163 markers of IR activation receptor (IGF-1R) based on embryonic stem cell differentiation studies [10].

In this paper, the in vitro and in vivo properties of the MNTF-33 derived GM6, a six-amino acid part of the endogenous motor neuron trophic factor is described, summarizing its potential as a new peptide therapeutic for ALS. Peptide therapeutics have several advantages being highly selective and efficacious and are well tolerated with good safety profiles [11]. The challenges with peptide therapeutics are their impaired cell permeability and their stability in plasma that has to be improved [11]. Therefore, the biomimetic properties of GM6 have been measured by HPLC using chemically bonded protein and phospholipid stationary phases [12]. The models developed for small molecules [13] have been applied to estimate the in vivo PK profile of GM6 with special emphasis on the expected free tissue concentration. GM6 has been found to have good drug-like properties with an estimated good cellular accumulation and tissue partition as has been described previously [14].

\section{Discovery of GM6}

GENERVON Biopharmaceuticals LLC has discovered and patented [9] several peptides. The hypothesis is that the master regulator for the differentiation of the human nervous system must be endogenous and the 3D structure should not be modified in order to be able to bind to a specific receptor. The natural motoneuron trophic factor (MNTF) consists of 33 amino acids. It's smaller analogue GM6 which is a partial sequence of MNTF and is a 6 amino acid cationic peptide. This potential drug modelled on an endogenous multi-target embryonic stage protein isolated from the nervous system. It was synthesized and then tested In the SOD1/G93A transgenic mouse model for ALS, GM6 delayed disease onset (27\%) and significantly extended the survival (30\%) and improved motor function (41- $43 \%$ ) [15].

\section{Experimental}

\section{Physicochemical and biomimetic property measurements - estimating in vivo distribution of GM6}

All high-performance liquid chromatography (HPLC) measurement were performed on an Agilent 100 equipped with an ultraviolet diode array detector (UV-DAD). The reversed phase fast gradient retention times have been measured using a Gemini NT C-18 column with the dimensions of $50 \times 3 \mathrm{~mm}$, particle size $5 \mu \mathrm{M}$ with a $110 \AA$ pore size (Phenomenex UK).

The starting mobile phase was $\mathrm{pH} 2.60 .01 \mathrm{M}$ formic acid for low $\mathrm{pH}$ lipophilicity, $50 \mathrm{mM}$ ammonium acetate buffers with $\mathrm{pH}$ adjusted to 7.4 and 10.5 by concentrated ammonia solution for neutral and basic $\mathrm{pH}$ lipophilicity measurements respectively. All reagents were HPLC grade obtained from Honeywell/Sigma-Aldrich, Germany. A $1.00 \mathrm{ml} / \mathrm{min}$ flow rate was applied. The acetonitrile gradient was 
from 0 to $100 \%$ from 0 to $3.5 \mathrm{~min}$ then $100 \%$ acetonitrile was kept until $4.5 \mathrm{~min}$ and then returned to $0 \%$ at $4.7 \mathrm{~min}$. The run time was $6 \mathrm{~min}$. HPLC grade water and acetonitrile were obtained from Rathburn Chemicals Ltd, Walkerburn, UK. The gradient retention times were standardized using the Valko test mixture and the chromatographic hydrophobicity index (CHI) values as described previously $[13,16]$. The $\mathrm{CHI}$ values were converted to the octanol/water $\log D$ scale using Equation 1.

$\mathrm{CHI} \log D=0.0525 \mathrm{CHI}-1.467$

In this way, the peptides lipophilicity can be compared to their octanol/water lipophilicity.

Measurements of membrane binding using immobilized artificial membrane (IAM) chromatography

For the measurements of GM6 interactions with phospholipids, the gradient retention times have been measured using an IAM.PC.DD2 $100 \times 4.6 \mathrm{~mm}$ column with $10 \mu \mathrm{M}$ diameter and $300 \AA$ pore size particles. The starting mobile phase was $50 \mathrm{mM}$ ammonium acetate with the $\mathrm{pH}$ adjusted to 7.4. The acetonitrile gradient was applied from 0 to $90 \%$ from 0 to $4.75 \mathrm{~min}$ and kept at $90 \%$ until $5.25 \mathrm{~min}$. From 5.25 to 5.5 min the acetonitrile concentration was dropped to $0 \%$. The mobile phase flow rate was $1.5 \mathrm{ml} / \mathrm{min}$ and the run time was $6 \mathrm{~min}$. The retention times were standardized using the IAM calibration mixture as described previously [17] (Equation 2).

$\log K_{\text {IAM }}=0.29 * \exp (0.046 \mathrm{CHI}$ IAM +0.42$)+0.70$

The log $K_{\text {IAM }}$ values express the membrane partition comparable to the octanol/water lipophilicity.

Protein binding measurements using biomimetic protein stationary phases

The peptides interactions with human serum albumin (HSA) and alpha-1-acid-glycoprotein (AGP) have been measured using commercially available chemically bonded HSA (Chiralpak-HSA) and AGP (ChiralpakAGP) HPLC columns with the dimensions of $50 \times 3 \mathrm{~mm}$ with a $5 \mu \mathrm{M}$ particle size obtained from HiChrom Ltd, Reading, UK. The mobile phase was $50 \mathrm{mM}$ ammonium acetate with the $\mathrm{pH}$ adjusted to 7.4. An isopropanol gradient (HPLC grade, Rathburn Chemicals, Walkerburn, Uk) was used from 0 to $35 \%$ from 0 to $3.5 \mathrm{~min}$ with a flow rate of $1.5 \mathrm{ml} / \mathrm{min}$. The $35 \%$ iso-propanol mobile phase was run till $4.5 \mathrm{~min}$ and then back to $0 \%$ within $0.2 \mathrm{~min}$. The run time was $6 \mathrm{~min}$ to allow re-equilibrate of the protein phase with the buffer. The retention times were standardized using the calibration set of compounds as described previously. Using the slope and intercept values from the calibration line the logarithmic retention times were converted to log $k$ values that can be converted to $\%$ binding values (\% HSA and $\%$ AGP) using Equation 3 [18].

$$
\% \text { bound }=\frac{101 \cdot 10^{\log k}}{1+10^{\log k}}
$$

In order to be able to estimate the in vivo distribution, cell penetration, cell membrane affinity and plasma protein binding biomimetic HPLC characterization of GM6 were carried out using the methods described earlier $[13,14,19]$. The in vivo distribution behaviour of GM6 has been estimated on C-18, IAM, HSA and AGP stationary phases based on the measured generic gradient retention times described above. The model equations with the source references are listed in Table 1.

\section{Stability measurements in human plasma}

The plasma stability of GM6 has been investigated by using a standard HPLC/MS/MS procedure by Frontage Laboratory Inc (Malvern, PA, USA). This bioanalytical laboratory measured the concentration of 
GM6 in human plasma using K2EDTA as the anticoagulant. The system was calibrated using GM6 in the concentration range of $250 \mathrm{ng} / \mathrm{ml}$ to $50 \mathrm{mg} / \mathrm{ml}$. The mean GM6 concentration was measured at 10, 15, 30 and 60 min time points after incubation. A considerable difference was observed in the mean concentration of the peptide GM6 within each individual lot of whole human blood at times 10, 15, 30 and 60 min compared with the 1 minute time point, which indicated that in the absence of stabilizer, GM6 is not stable in fresh human blood. It is worth mentioning that in this study only the intact GM6 molecule concentration was monitored.

Table 1. The model equations used to derive estimated in vivo distribution ( $\log V_{\mathrm{dss}}=$ logarithm of the volume of distribution, $\log V_{\mathrm{du}}=$ logarithm of the unbound volume of distribution), drug efficiency $\left(\mathrm{DE}_{\max }\right)$, brain tissue binding $\left(\log k_{\mathrm{BTB}}\right)$, fraction unbound in brain and plasma ( $\mathrm{fu}_{\mathrm{BTB}}$ and $\left.\mathrm{fu} \mathrm{PPB}_{\mathrm{PPB}}\right)$, brain to plasma ratio $\left(k_{\mathrm{bb}}\right)$ and cell partition log $\left.K_{\text {pcell }}\right)$.

\begin{tabular}{|c|c|}
\hline $\log K_{\mathrm{IAM}}[17]$ & $\left.=0.29 * \mathrm{e}^{(0.026 \mathrm{CH}(\mathrm{IAM})+0.42}\right)+0.7$ \\
\hline $\log k_{\mathrm{IAM}}[17]$ & $=0.046^{*} \mathrm{CHI}(\mathrm{IAM})+0.42$ \\
\hline $\log K_{\mathrm{HSA}}[17]$ & $=\mathrm{e}^{\log k(\mathrm{HSA})}$ \\
\hline $\log k_{\mathrm{HSA}}[18]$ & $=\log (\% H S A b o u n d /(101-\% H S A$ bound $))$ \\
\hline Estimated log $V_{\mathrm{dss}}[17]$ & $=0.44 * \log K_{\mathrm{IAM}}-0.22 * \log K_{\mathrm{HSA}}-0.62$ \\
\hline Estimated $\log V_{\mathrm{du}}[20]$ & $=0.23 * \log K_{\mathrm{HSA}}+0.43 * \log K_{\mathrm{IAM}}-0.72$ \\
\hline $\mathrm{DE}_{\max }[21]$ & $=100 / V_{\mathrm{du}}$ \\
\hline $\log k_{\mathrm{BTB}}[22]$ & $=1.29 * \log k_{\mathrm{IAM}}+1.03 * \log k_{\mathrm{HSA}}-2.37$ \\
\hline $\log k_{\mathrm{PPB}}[22]$ & $=0.98 * \log k_{H S A}+0.19 * \log k_{A G P}+0.031 * \mathrm{CHI} \log D_{7.4}-0.20$ \\
\hline \%BTB [22] & $=100 * 10^{\log k_{\mathrm{BTB}}} /\left(1+10^{\log k_{\mathrm{BTB}}}\right)$ \\
\hline \%PPB [22] & $=100 * 10^{\log k_{\mathrm{PPB}}} /\left(1+10^{\log k_{\mathrm{PPB}}}\right)$ \\
\hline fu BTB and PPB [22] & $=(100-\%$ BTB $) / 100$ and $(100-\%$ PPB $) / 100$ \\
\hline$K_{\mathrm{bb}}[22]$ & $=f u_{P P B} / f u_{B T B}$ \\
\hline $\log K_{\text {pcell }}[23]$ & $=1.1 * \log k_{\text {IAM }}-1.9$ \\
\hline
\end{tabular}

In vitro clearance and estimated PK profile of GM6

In order to estimate the expected PK profile of GM6 in human the in vitro microsomal stability has been measured. Eurofins Pharma Discovery Services Cerep Inc (France) carried out in vitro clearance measurements on GM6 using human liver microsomes. The in vitro clearance data so obtained can be used to estimate the in vivo clearance and half-life using a well-stirred model [24]. A comparative study was carried out using GM6 and Riluzole, an FDA approved drug for ALS. The purpose of the study was to test whether there was any interaction between GM6 and Riluzole in the intrinsic clearance assay using human liver microsomes.

A pool of 50 mixed gender human liver microsomes was used with a final microsomal concentration 0.1 $\mathrm{mg} / \mathrm{ml}$. The test compounds were dissolved to a $0.1 \mu \mathrm{M}$ concentration in $0.01 \% \mathrm{DMSO}, 0.25 \%$ acetonitrile and $0.25 \%$ methanol. The test compounds were pre-incubated with the pooled liver microsomes in phosphate buffer and shaken for $5 \mathrm{~min}$ at 37 centigrade. The reaction was initiated by an NADPHgenerating system and incubated for 0, 15, 30, 45 and $60 \mathrm{~min}$. The reaction was then stopped by transferring the incubation mixture to an acetonitrile/methanol mixture. Samples were then mixed and centrifuged and the supernatant analysed by HPLC-MS/MS. The compound remaining after incubation was 
calculated from the peak area obtained for each sample time point relative to the zero-time point. The half-life was calculated from the slope of the initial linear range of the logarithmic curve of the compound remaining intact (\%) versus time, assuming first-order kinetics. The intrinsic clearance was calculated using Equation (4).

$$
C l_{\text {int }}(\mu \mathrm{l} / \mathrm{min} / \mathrm{mg} \text { protein })=\frac{0.693}{t_{1 / 2} \cdot \text { Protein conc. }}
$$

\section{Quantitative immunofluorescence imaging}

Phenovista Biosciences LLC (San Diego, CA, USA) provided several phenotypic assays on four different cell types treated with GM6 in order to reveal the presence of, and reaction to, GM6 in these cells. The primary goal of the study was to identify antibody-based markers that could be used successfully in quantitative imaging-based analysis and to determine if the application of GM6 caused quantifiable effects on signalling pathways identified by a series of antibody markers. Four human cell types were used:

1. iPS-derived iCell hepatocytes 2.0 (CDI PHC-100-020-001) (cultured 10 days in manufacturers suggested media then treated with GM6),

2. human brain microvascular endothelial cells [HBMVECs] (iX Cells 10HU-051) (cultured 2 days in manufacturers suggested media then treated with GM6),

3. ntera2 differentiated cells (ATCC \#CRL-1973) (differentiated for 21 days in $10 \mu \mathrm{M}$ retinoic acid (RA), $0.5 \mathrm{mM}$ IBMX and $1 \mathrm{mM}$ 8-Br-CAMP then treated with GM6),

4. iPS-derived iCell GABAergic neurons (CDI NRC-100-010-001) (cultured 7 days in manufacturers suggested media then treated with GM6).

All cells were cultured and treated in high density 384 well microplates before fixation, immunofluorescence staining and analysis using automated quantitative microscopy. The aim of the analysis was to determine uptake and downstream signalling effects of GM6 via assessment of the marker levels in each cell type under each treatment condition. Due to the unknown distribution and response of the twelve unique markers selected, a general-purpose image analysis strategy was taken whereby changes in cellular intensity for each marker would be quantified.

Cells were seeded in 384 well plates (Greiner) in culture media according to manufacturer's guidelines and where applicable differentiated before treatment with GM6 for 15 minutes, 2 or 4 hours. Treatments were performed via the addition of a $10 x$ solution as $10 \%$ of the final culture volume (e.g. $2.2 \mu \mathrm{l}$ of $10 \mathrm{mM}$ $\mathrm{GM} 6$ added to $20 \mu \mathrm{l}$ culture volume for a final concentration of $1 \mathrm{mM} \mathrm{GM6).} \mathrm{After} \mathrm{treatment} \mathrm{the} \mathrm{cells} \mathrm{were}$ fixed in $4 \%$ formaldehyde for 15 minutes, washed in PBS containing $0.3 \%$ Triton X-100 for 5 minutes at room temperature then blocked in PBS containing either $5 \%$ goat serum or $5 \%$ donkey serum (depending on primary antibody host used), $1 \% \mathrm{BSA}$, and $0.2 \%$ fish gelatin overnight at $4{ }^{\circ} \mathrm{C}$. Primary antibody diluted in blocking buffer was incubated at $4{ }^{\circ} \mathrm{C}$ overnight followed by three washes in PBS then fluorescentlyconjugated secondary antibodies (1:500 dilution, and Hoechst $33342(10 \mu \mathrm{g} / \mathrm{ml}$ final) added in PBS and incubated at room temperature for 1 hour before washing in PBS three times and storage at $4{ }^{\circ} \mathrm{C}$.

Twelve unique markers were assessed in combination with the nuclear marker Hoechst 33342 in six staining sets (aka palettes); chicken anti-GM6 (Genervon), rabbit anti-insulin receptor (Abcam \#ab131238), goat anti-SOD1 (Abcam \#ab62800), mouse anti-insulin receptor beta (Abcam \#ab983), rabbit anti-phospho Tau S356 (Abcam \#ab75603), mouse anti-PI3Kp85 (Abcam \#ab86714), rabbit anti-TDP43 (ThermoScientific \#PA5-29949), rabbit anti IGF1 receptor beta (Cell Signalling \#9750), mouse anti-tau (Synaptic Systems 
\#314011), rabbit anti-phospho tau T231 (Abcam \#ab151559), mouse anti-IGF1 receptor (Abcam ab16890) and rabbit anti-phospho insulin recptor beta Y972 (Abcam 5678).

Imaging was performed using a ThermoScientific CX7 automated fluorescence microscope. Images were collected using filter sets appropriate for the fluorescently-conjugated secondary antibodies and images analysed using the Compartmental Analysis (ThermoScientific) algorithm as part of the HCS studio software package. Data were processed using Microsoft Excel to calculate mean, standard deviation and strictly standardized mean difference (SSMD) scores to assess statistical significance versus control treatments.

In vivo brain penetration studies

C57BL6 mice were injected with a single bolus IV tail vein injection of GM6 at 0.2 and $2.0 \mathrm{mg} / \mathrm{kg}$. At four hours, the animals were sacrificed, and half of the brain was frozen for ELISA analysis. The detailed experimental process has been published previously [25].

\section{Results and Discussion}

Physicochemical and biomimetic properties of GM6

It is important to determine how GM6 behaves in vivo and what are its physicochemical and drug-like properties and can it's in vivo distribution and pharmacokinetic behaviour be predicted. Similar to other peptide therapeutics it was expected that GM6 would not be stable in plasma, so measurement of the traditionally used plasma vs. time PK profile would not be feasible. Therefore, the protein and phospholipid binding of GM6 was assessed using biomimetic HPLC experiments. The results of the measurements of protein and phospholipid binding of GM6 are listed in Table 2, including the estimated in vivo distribution properties of GM6 using the model equations listed in Table 1.

It can be seen that due to the two arginine amino acids in the sequence GM6 is positively charged at physiological pH. Therefore, it's binding to alpha-1-acid glycoprotein (AGP) and Immobilized Artificial Membrane (IAM) is very strong. The total plasma protein binding is expected to be $31 \%$. However, due to the strong IAM binding, it is very likely that GM6 also partitions quickly into the blood cells. The volume of distribution was estimated from the difference of binding of GM6 to albumin and IAM according to the model published for marketed drugs [17] that was $7.8 \mathrm{~L} / \mathrm{kg}$ using the equation listed in Table 1.

The brain tissue binding of GM6 is estimated to be $58.3 \%$ which is higher than the plasma protein binding suggesting higher total brain concentration than total plasma concentration. The brain to plasma total concentration ratio is expected to be 1.65. Due to the strong IAM binding, the estimated intracellular concentration is approximately 5 times higher than the extracellular concentration using the models obtained with HELA cell [23]. The drug efficiency which is defined as the free bio-phase concentration relative to the dose [26] is over $8 \%$ which is much above the expected $1 \%$ that is characteristic of the marketed drugs.

From the biomimetic models only, the total brain to plasma concentration ratio can be estimated based on the difference between the brain tissue and the plasma protein binding as shown in Table 1.

Figures 1 and 2 show that GM6 has good drug-like properties similar to marketed small molecule drugs.

GM6 is well positioned in the middle of the marketed drugs based on the plot of the estimated brain to blood ratio $\left(\log k_{\mathrm{BB}}\right)$ and steady-state volume of distribution ( $\left.\log V_{\mathrm{dss}}\right)$.

It was found that $70 \%$ of GM6 was stable in human liver microsomes after $60 \mathrm{~min}$. The addition of 
Riluzole to the liver microsomes did not change the clearance of GM6 nor the Riluzole clearance, suggesting no drug-drug interactions. Further studies will be needed to estimate the renal clearance of peptides which is a more typical route of elimination [27].

Table 2. The measured biomimetic properties of GM6 using published models for small molecule marketed drugs [22]

\begin{tabular}{|c|c|c|}
\hline Property & Value & Explanation \\
\hline$\%$ bound HSA & 22.90 & Albumin binding \\
\hline$\%$ bound AGP & 45.06 & Glycoprotein binding \\
\hline Calc \% PPB & 31.40 & Plasma protein binding \\
\hline Calc \% Blood & 23.60 & Blood binding \\
\hline $\mathrm{CHI} \log D_{2}$ & -3.38 & Lipophilicity at pH 2 \\
\hline $\mathrm{CHI} \log D_{7.4}$ & -0.16 & Lipophilicity at pH 7.4 \\
\hline $\mathrm{CHI} \log D_{10.5}$ & 0.20 & Lipophilicity at pH 10.6 \\
\hline $\mathrm{CHI} \log P$ & 0.20 & Neutral form lipophilicity \\
\hline IAM & 42.5 & Phospholipid binding \\
\hline CAD-likeness & 49.4 & Phospholipidotic potential \\
\hline est log BB & 0.22 & Brain to blood ratio (log) \\
\hline Brain to plasma & 1.65 & Brain to plasma ratio \\
\hline $\log V_{d}$ & 0.89 & log Volume of distribution \\
\hline$V_{\mathrm{d}} \mathrm{L} / \mathrm{kg}$ & 7.83 & Volume of distribution \\
\hline $\log V_{\mathrm{du}}$ & 1.06 & log Unbound volume of distribution \\
\hline$V_{\mathrm{du}}$ & 11.5 & The unbound volume of distribution \\
\hline DEmax\% & 8.74 & max Drug efficiency \\
\hline MW & 799.00 & Molecular weight \\
\hline $\log k_{\text {Brain }}$ & 0.15 & Brain tissue partition \\
\hline$\%$ BTB & 58.3 & Brain tissue binding \\
\hline $\log k$ lung tissue & 0.51 & Lung tissue partition \\
\hline$\%$ lung tissue binding & 72.3 & Lung tissue binding \\
\hline $\log K_{\mathrm{p}}$ skin & -7.10 & log Skin partition \\
\hline $\log K_{\mathrm{p}}$ skin & -7.69 & Skin partition \\
\hline $\log K_{\mathrm{p}}$ cell & 0.71 & log cell partition \\
\hline$K_{\mathrm{p}}$ cell & 5.2 & Cell partition \\
\hline
\end{tabular}

It is well-known that plasma proteases can hydrolyse peptides rapidly and that special method should be applied to the plasma concentration determination [28,29]. GM6 showed 15 min half-life in vitro in human blood.

It has been also reported that some therapeutic peptides could not be detected in the plasma but they still had a long-lasting pharmacological effect [30]. There are various methods for increasing the plasma half-life of peptides such as replacing L-amino acids to D- amino acids, blocking the $\mathrm{N}$ or $\mathrm{C}$ terminals, conjugations to macromolecules like PEG and cyclisation [27]. Other approaches such as increasing the albumin binding are also used to improve the physicochemical and in vivo ADMET properties of peptides [31] in order to open up new modalities in drug discovery. With GM6, the idea was not to change the peptide structure but to remain close to the endogenous MNTF using the 6-amino acid sequence which was thought to be responsible for the neuroprotective effect. 


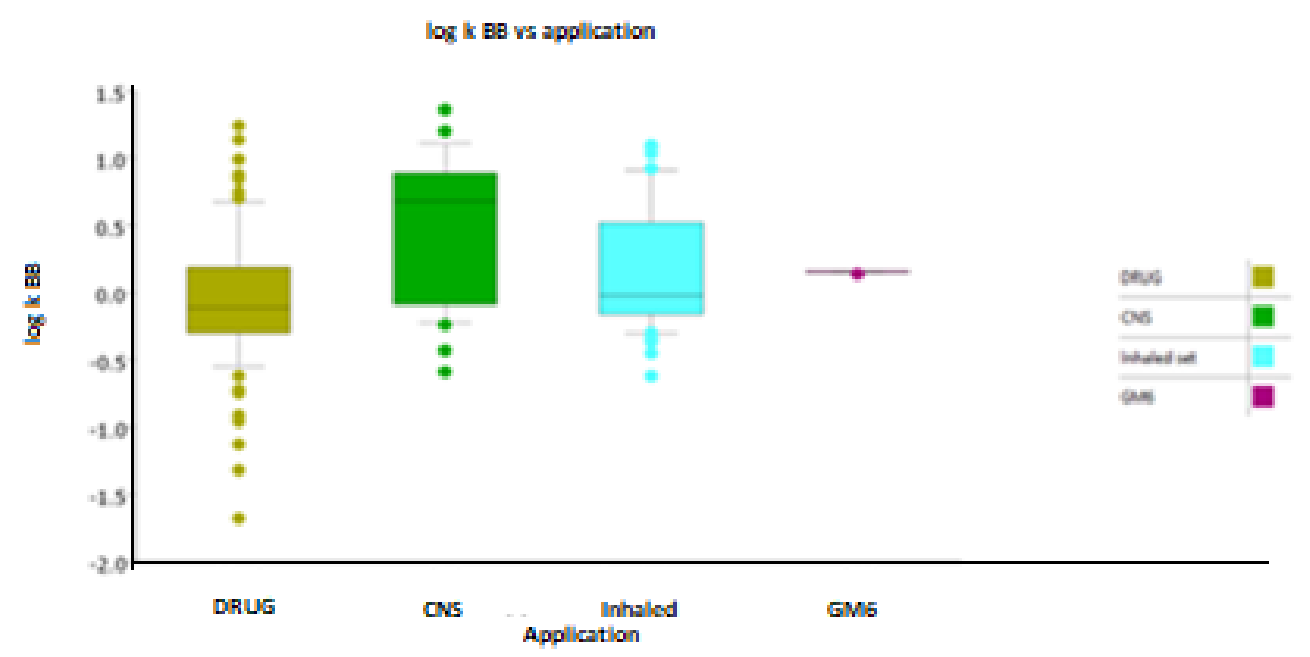

Figure 1. Boxplot of the average estimated $\log k_{\mathrm{BB}}$ values of marketed drugs, drugs that enter into the central nervous system (CNS) and inhaled drugs, as well as GM6. The estimated brain to plasma total concentration ratio was calculated using the equations given in Table 1.

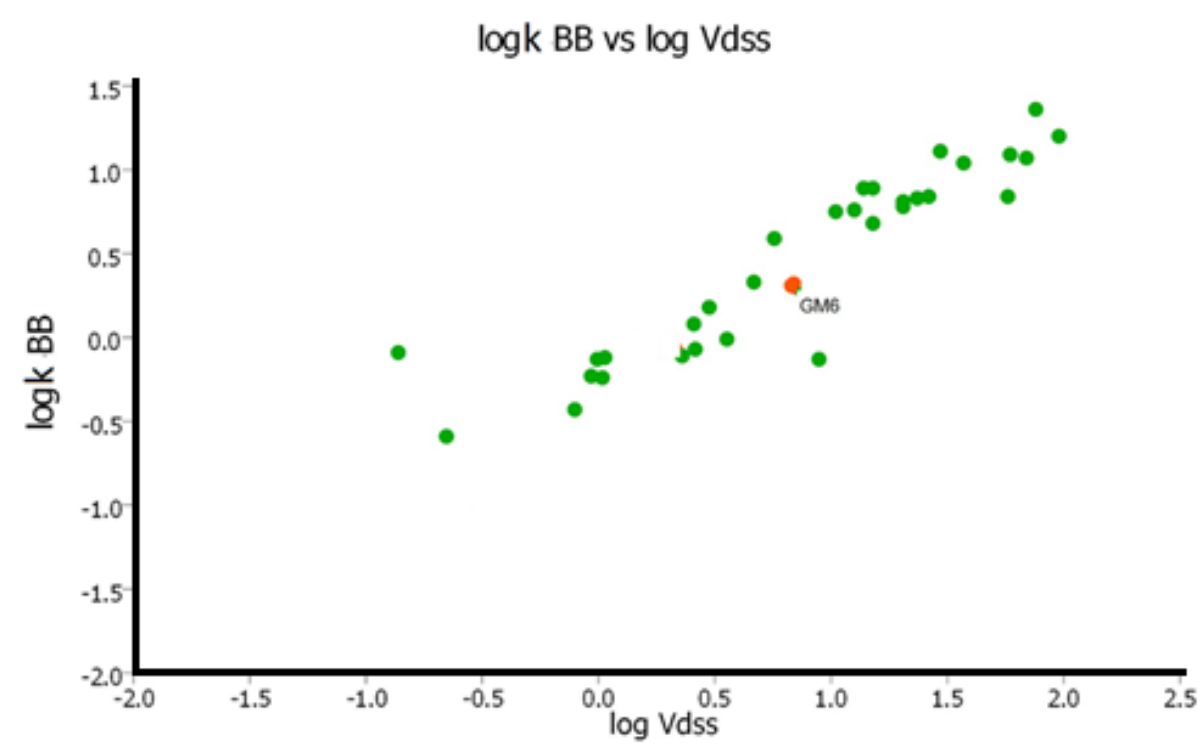

Figure 2. The plot of the estimated brain to blood ratio $\left(\log k_{\mathrm{BB}}\right)$ and the steady-state volume of distribution $\left(\log V_{\text {dss }}\right)$ of marketed drugs that enter into the CNS.

Based on the measured physicochemical and biomimetic properties listed in Table 2, it can be seen that GM6 is predicted to have high cell partition $\left(K_{\text {pcell }}=5.2\right)$ which means 5 times higher concentration inside the cells that outside the cells. The model has been developed using 300 compounds data that were equilibrated with HELA cells and measured the intracellular concentration [23]. HELA cells do not contain active transporters, so it can be assumed that this is the passive partition of GM6 into cells.

\section{Quantitative immunofluorescence imaging}

The results of the quantitative imaging experiments gave a very good indication that GM6 associates with cells well, especially to iPS-derived neurons. GM6 demonstrated quantifiable labelling of cellular structures in all of the cell types tested with chicken anti-GM6 (Genervon). While it is difficult to determine whether GM6 was restricted to the surface of the cells that it interacted with or was internalized or degraded, based on the time and dose-dependent effects on markers, it did suggest that GM6 was able to elicit cellular responses that could be a result of cell surface binding, via uptake or via a combination of 
both processes. It was found that an increase in GM6 abundance in all cell types could be quantified. The response of GM6 treatment on the GABAergic neurons was much more significant than on the hepatocytes, which suggested a specific affinity of GM6 to tissues of the nervous system and less so to the liver. Based on the study we can hypothesize that this cationic peptide, GM6, crosses the cell membrane and enters the cytoplasm. Figure 3 shows representative immunofluorescence images obtained in GABA neuron cells after treatment of GM6.

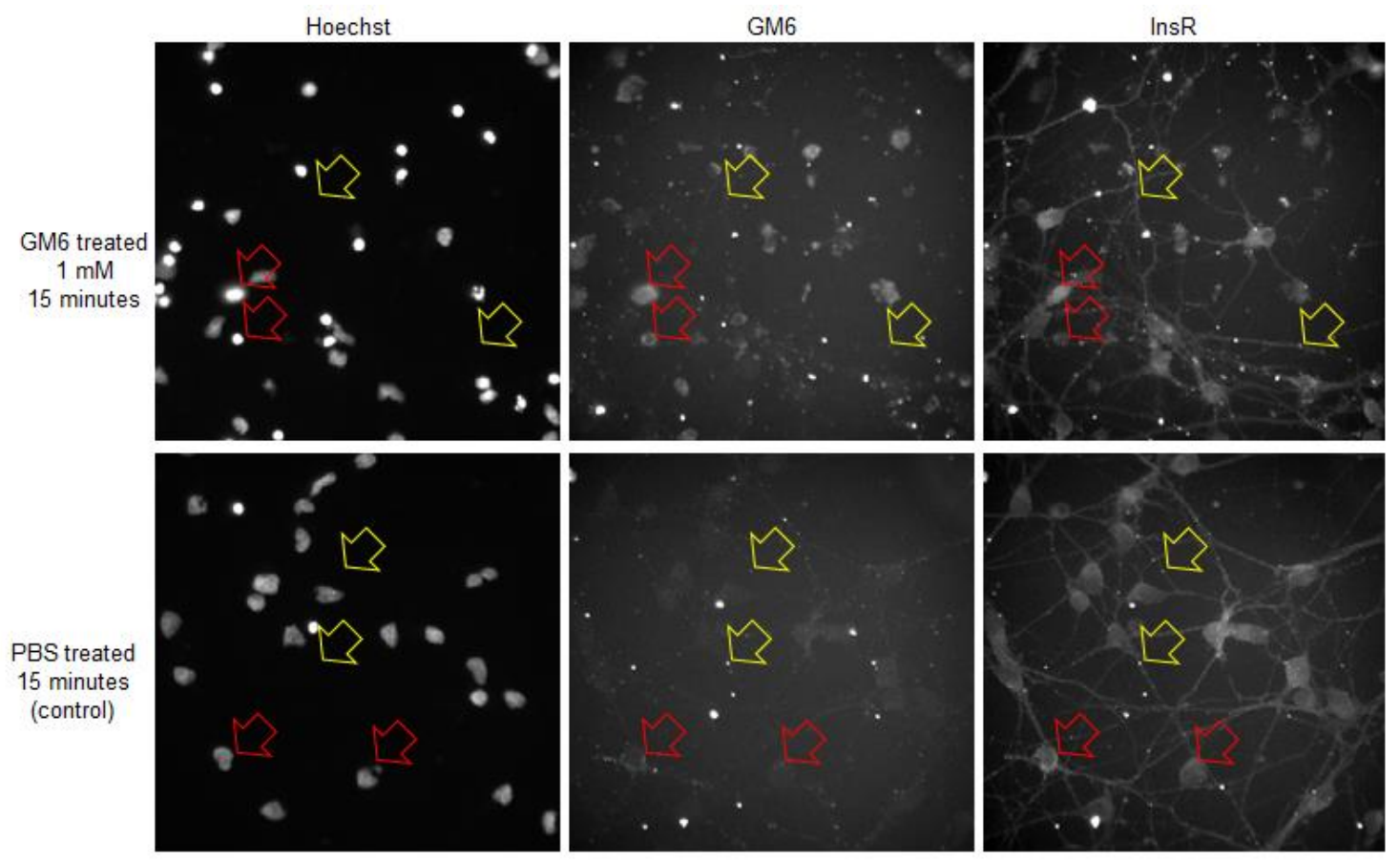

Figure 3. Immunofluorescence images obtained after treating the GABA neuron cells with $1 \mathrm{mM}$ GM6 or PBSonly control. (Top row shows staining of human iPS-derived GABAergic neurons treated with $1 \mathrm{mM}$ GM6 for 15 minutes while the bottom row shows control PBS-treated neurons. Leftmost panels show Hoechst staining that identifies neuronal nuclei. Panels in the middle column show detection of GM6 in the same cells using an anti-GM6 antibody while columns to the right show corresponding insulin receptor staining intensity, which has been included to show the location of the cell bodies and neurites. Red arrows show GM6 in the cell body

while yellow arrows show GM6 puncta localized to neurites. In some cases, staining of GM6 and InsR is coincident.)

Results of the in vivo brain penetration studies in mice

ELISA assays with brain homogenate supernatant detected GM6 at statistically significant levels, at all doses, compared to the control $(p=0.0001)$ as shown in Figure 4 . The vehicle group has some detection of GM6 $(0.4050 \mu \mathrm{M})$. The $0.2 \mathrm{mg} / \mathrm{kg}$ group has $1.76 \mu \mathrm{M}$, the $2.0 \mathrm{mg} / \mathrm{kg}$ group has $12.92 \mu \mathrm{M}$. If we subtract the GM6 in the vehicle group, the net increase from injection of GM6 for the $0.2 \mathrm{mg} / \mathrm{kg}$ is $1.36 \mu \mathrm{M}$, for the 2.0 $\mathrm{mg} / \mathrm{kg}$ is $12.52 \mu \mathrm{M}$. The net increase of GM6 detected is almost proportional.

These results support the prediction that GM6 can partition into tissues based on its large volume of distribution and that has also a good brain penetration [25] (brain to plasma ratio was estimated as 1.65 as indicated in Table 2).

\section{Estimated PK profile of GM6}

In order to estimate the dose we have calculated the minimum effective concentration of GM6 obtained from the in vivo mouse experiment described in more detail later. The in vivo results showed that 
a $20 \mathrm{mg} / \mathrm{kg}$ dose of GM6 was efficacious in the mouse. Using the 12.3 factor from mouse to human according to the FDA guidelines [32], the dose was estimated to be $1.6 \mathrm{mg} / \mathrm{kg}$ in human. As the drug efficiency of the compound is around $8 \%$ it means that only $8 \%$ of the administered dose is available as free. That is equivalent to $0.08 \cdot 1.6 \mathrm{mg} / \mathrm{kg}$, which is $0.13 \mathrm{mg} / \mathrm{kg}$ which equals $1.3 \cdot 10^{-4} \mathrm{~g} / \mathrm{kg}$. Taking into account the 799 Da molecular weight of GM6 it is equivalent to a $1.6 \cdot 10^{-7} \mathrm{M}$ concentration. It means that the pIC50 or in other words the minimum effective concentration (MEC) of the peptide is estimated to be around $1.6 \cdot 10^{-7} \mathrm{M}$. The dose can be estimated from the pIC50 and the drug efficiency as it has been described earlier [21]. This dose estimation refers to a steady state and does not take into account the elimination rate and dosing frequency.

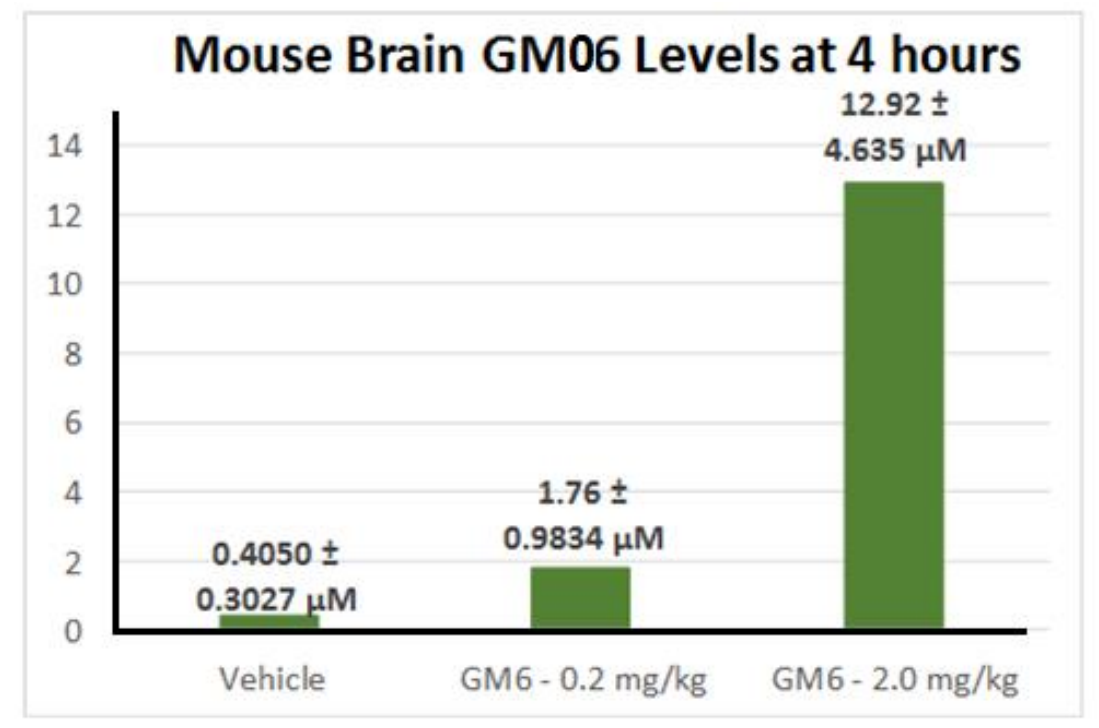

Figure 4. Detection GM6 in mouse brain after four hours of the treatment by ELISA analysis.

Using the one-compartment model [33] and a $132.5 \mathrm{mg}$ dose for an average $70 \mathrm{~kg}$ human, a hypothetical PK profile can be calculated using the parameters listed in Table 3 based on equation (5).

$$
\text { Dose }(\mathrm{mg} / \mathrm{kg} / \mathrm{day})=\frac{\frac{24}{\tau} \mathrm{MEC} \cdot V_{d s s}\left(\exp \left(k_{e l} \tau\right)-1\right)}{F}
$$

Where $\tau$ is the dosing interval, $k_{\mathrm{el}}$ is the elimination rate calculated from the in vitro clearance (Equation (4)), $V_{\text {dss }}$ is the steady-state volume of distribution, $F$ is the bioavailability considered 1 and the absorption rate considered fast after IV administration.

The intrinsic clearance (Clint) for GM6 in human microsome assays was determined to be $<115.5$ $\mu \mathrm{g} / \mathrm{mL} / \mathrm{min} / \mathrm{mg}$. To convert the Clint data from $115 \mathrm{ug} / \mathrm{min} / \mathrm{mg}$ human liver microsomes to $\mathrm{mL} / \mathrm{min} / \mathrm{kg}$ we used the human scaling factor of $\mathrm{mg}$ protein in liver: $115 \times 39.7=4565.5 \mu \mathrm{g} / \mathrm{min} / \mathrm{g}$ liver which is equivalent to $4565 / 1000=4.565 \mathrm{ml} / \mathrm{min} / \mathrm{g}$ liver. This value should go to the well-stirred model (WSM) to estimate the human whole-body clearance $\left(k_{\mathrm{el}}\right)$. Using the WSM model it is equivalent to $15.5 \mathrm{ml} / \mathrm{min} / \mathrm{kg}$ (which is equivalent to $86 \%$ of the liver blood flow) as the clearance of GM6 which is relatively rapid. It is supposed that $\mathrm{GM} 6$ being an endogenous neuropeptide with two positive charges and relatively small $(M W=799)$ the liver microsomes provide the major elimination route. It is also important to note that GM6 has a large estimated volume of distribution (over $7.8 \mathrm{~L} / \mathrm{kg}$ ) unlike other peptides and it binds to phospholipid very strongly (CHI IAM value is above 42). In a recent review [34] about the pharmacokinetic studies of protein, drugs raise the question whether the blood concentration is relevant or not to pharmacodynamics with the emphasis on the tissue concentration. 
Table 3. The parameters used in estimating the PK profile of GM6 in human after 132 $\mathrm{mg}$ iv dose.

\begin{tabular}{|l|c|}
\hline Body Weight $(\mathrm{kg})$ & 70 \\
\hline Dosing interval $(\tau)$ & 1 \\
\hline Dose $(\mathrm{mg} /$ day) & 132.5 \\
\hline Dose $(\mathrm{mg} / \mathrm{Kg})$ & 1.89 \\
\hline$V_{\mathrm{d}}(\mathrm{L} / \mathrm{Kg})$ & 7.8 \\
\hline $\mathrm{CL}(\mathrm{mL} / \mathrm{min} / \mathrm{Kg})$ & 15.5 \\
\hline $\mathrm{CL}(\mathrm{L} / \mathrm{h} / \mathrm{Kg}))$ & 0.93 \\
\hline $\mathrm{CL}(\mathrm{mL} / \mathrm{min})$ & 1085.0 \\
\hline $\mathrm{CL} / F(\mathrm{~mL} / \mathrm{min})$ & 1085.0 \\
\hline $\mathrm{CL} / F(\mathrm{~L} / \mathrm{min})$ & 1.085 \\
\hline fu blood $(\%)$ & 68.60 \\
\hline$T_{\max }(\mathrm{h})$ & 0.8 \\
\hline$C_{\max }(\mathrm{ng} / \mathrm{mL})$ & 222 \\
\hline$K_{\mathrm{e}}(1 / \mathrm{h})$ & 0.12 \\
\hline$t_{1 / 2}(\mathrm{~h})$ & 5.8 \\
\hline AUC $(\mathrm{ng} / \mathrm{ml}$ *h) & 2037 \\
\hline$V_{\text {du }}(\mathrm{L} / \mathrm{Kg})$ & 11.4 \\
\hline DRUGeff MAX $(\%)$ & 8.8 \\
\hline DRUGeff Blood $C_{\max }$ u (\%) & 8.03 \\
\hline Compound Information & $\mathrm{GM6}$ \\
\hline Molecular weight & 799 \\
\hline $\mathrm{pXC50}(\mathrm{based}$ on in vivo efficacious concentration) & 7 \\
\hline Conc (nM) & 100.00 \\
\hline Total Conc (nM) & 145.77 \\
\hline Conc $(\mathrm{ng} / \mathrm{mL})$ & 79.90 \\
\hline Total Conc $(\mathrm{ng} / \mathrm{mL})$ & 116.47 \\
\hline
\end{tabular}

Equation 5 describes the hypothetical plasma concentration vs time profile of drugs. As GM6 has a short half-life in plasma but it was detected in brain tissue after 4 hours, instead of the total plasma concentration vs time profile the free concentration-time profile was calculated that is referred to tissues. The estimated half-life in tissues including only the microsomal clearance was calculated as $5.8 \mathrm{~h}$.

This PK profile would have been very difficult to measure in vivo due to the technical difficulties of detecting GM6 in human blood. At this stage, the renal clearance of the compound had not been tested. Even if the elimination rate is double ( $30 \mathrm{ml} / \mathrm{min} / \mathrm{kg}$ ) due to renal clearance of GM6 we can expect 3 hours half-life in the tissues when calculated using equation 5 .

\section{Conclusions}

In this paper, results have been shown and discussed for a 6-amino acid fragment (GM6) of the endogenous motor neuron trophic factor (MNTF) containing 33 amino acids and further discussed as a potential peptide therapeutic for amyotrophic lateral sclerosis (ALS). The physicochemical properties of GM6 were measured using biomimetic HPLC membrane and protein binding. GM6 showed very good druglike properties. Due to the two positively charged arginine amino acids and based on the measured phospholipid and protein binding, GM6 has been predicted to have very strong tissue partition with an estimated volume of distribution of $7.8 \mathrm{~L} / \mathrm{kg}$. It was also predicted that it partitions into cells $\left(K_{\text {pcell }}=5.2\right)$ and has a good brain to plasma ratio (1.65). Studies in mice show penetration of the GM6 into the brain following a bolus iv injection with the drug still present after four hours. The concentration of GM6 
decreased rapidly in blood suggesting a high affinity for target sites in the brain and/or active uptake into brain cells and GABA-ergic neuron cells as shown by immunofluorescence imaging studies. The estimated free tissue concentration - time profile based on only microsomal elimination showed approximately 6 hours half-life. The in vivo beneficial effects of GM6 are longer lasting. This would indicate that it probably provokes a cascade of biochemical events, after initial binding, and that influences multiple pathways that promote neuron regeneration.

\section{References}

[1] S. Przedborski, H. Mitsumoto, L.P. Rowland. Recent advances in amyotrophic lateral sclerosis research. Curr. Neurol. Neurosci. Rep. 3 (2003) 70-77.

[2] M.C. Kiernan, S. Vučić, B.S. Cheah, M.R. Turner, A. Eisen, O. Hardiman, J.R. Burrel, M.C. Zoing. Amyotrophic lateral sclerosis. Lancet (London, England) 377 (2011) 942-955.

[3] P. Pasinelli, R.H. Brown. Molecular biology of amyotrophic lateral sclerosis: Insights from genetics. Nat. Rev. Neurosci. 7 (2006) 710-723.

[4] R.E. Ellis, J.Y. Yuan, H.R. Horvitz. Mechanisms and functions of cell death. Annu. Rev. Cell Biol. 7 (1991) 663-698.

[5] E.T. Reyes, O.H. Perurena, B.W. Festoff, R. Jorgensen, W.V Moore. Insulin resistance in amyotrophic lateral sclerosis. J. Neurol. Sci. 63 (1984) 317-324.

[6] S. Cohen. Purification of a nerve-growth promoting protein from the mouse salivary gland and its neuro-cytotxic antiserum. Proc. Natl. Acad. Sci. U. S. A. 46 (1960) 302-311.

[7] I.A. Hendry, K. Stöckel, H. Thoenen, L.L. Iversen. The retrograde axonal transport of nerve growth factor. Brain Res. 68 (1974) 103-121.

[8] R.M. Wah. Polynucleotides encoding motoneurontrophic factors. US Patent 6309877.

[9] R.M.W. Chau, P.-Y.D.Ko. Hong Kong. MNTF peptides and compositions and methods of use. US Patent 7183373, (2007).

[10] D.M. Deshpande, D. A. Kerr, M. Ruxton, D.P.-Y. Ko. NMNTF differentiation and growth of stem cellso Title. US Patent No 8986676, (2015).

[11] K. Fosgerau, T. Hoffmann. Peptide therapeutics: Current status and future directions. Drug Discov. Today 20 (2015) 122-128.

[12] K.L. Valkó. Lipophilicity and biomimetic properties measured by HPLC to support drug discovery. J. Pharm. Biomed. Anal. 130 (2016) 35-54.

[13] K.L. Valko, S.P. Teague, C. Pidgeon. In vitro membrane binding and protein binding (IAM MB/PB technology) to estimate in vivo distribution: applications in early drug discovery. ADMET DMPK 5 (2017) 14-38.

[14] K. Valko, G. Ivanova-Berndt, P. Beswick, M. Kindey, D. Ko. Application of biomimetic HPLC to estimate lipophilicity, protein and phospholipid binding of potential peptide therapeutics. ADMET DMPK (2018) http://dx.doi.org/10.5599/admet.544.

[15] M.K. D. Ko. Methods of treating neuronal disorders using MNTF peptides and analogs. US Patent 8673852, (2014).

[16] K. Valko, C. Du, C. Bevan, D. Reynolds, M. Abraham. Rapid Method for the Estimation of Octanol / Water Partition Coefficient (Log Poct) from Gradient RP-HPLC Retention and a Hydrogen Bond Acidity Term (Sigma alpha2H). Curr. Med. Chem. 8 (2001) 1137-1146.

[17] F. Hollósy, K. Valkó, A. Hersey, S. Nunhuck, G. Kéri, C. Bevan. Estimation of volume of distribution in humans from high throughput HPLC-based measurements of human serum albumin binding and immobilized artificial membrane partitioning. J. Med. Chem. 49 (2006) 6958-6971.

[18] K. Valko, S. Nunhuck, C. Bevan, M.H. Abraham, D.P. Reynolds. Fast Gradient HPLC Method to Determine Compounds Binding to Human Serum Albumin. Relationships with Octanol/Water and Immobilized Artificial Membrane Lipophilicity. J. Pharm. Sci. 92 (2003) 2236-2248. 
[19] K. Valko. Lipophilicity and biomimetic properties measured by HPLC to support drug discovery. J. Pharm. Biomed. Anal. 130 (2016) 35-54.

[20] K.L. Valkó, S.B. Nunhuck, A.P. Hill. Estimating Unbound Volume of Distribution and Tissue Binding by in vitro HPLC-based Human Serum Albumin and Immobilized Artificial Artificial Membrane-Binding Measurements. J. Pharm. Sci. 100 (2011) 849-862.

[21] K. Valko, E. Chiarparin, S. Nunhuck, D. Montanari. In vitro measurement of drug efficiency index to aid early lead optimization. J. Pharm. Sci. 101 (2012) 4155-4169.

[22] K. Valko, Physicochemical and biomimetic properties in drug discovery - Chromatographic techniques for lead optimization. Wiley, Hoboken NJ (2014).

[23] L.J. Gordon, M. Allen, P. Artursson, M.M. Hann, B.J. Leavens, A. Mateus, S. Readshaw, K. Valko, G.J. Wayne, A. West. Direct Measurement of Intracellular Compound Concentration by RapidFire Mass Spectrometry Offers Insights into Cell Permeability. J. Biomol. Screen. 21 (2016) 156-164.

[24] A.B. Ahmad, P.N. Bennett, M. Rowland. Models of hepatic drug clearance: discrimination between the 'well stirred' and 'parallel-tube' models. J. Pharm. Pharmacol. 35 (1983) 219-224.

[25] J. Yu, H. Zhu, D. Ko, M.S. Kindy. Motoneuronotrophic factor analog GM6 reduces infarct volume and behavioral deficits following transient ischemia in the mouse. Brain Res. 1238 (2008) 143-153.

[26] S. Braggio, D. Montanari, T. Rossi, E. Ratti. Drug efficiency: a new concept to guide lead optimization programs towards the selection of better clinical candidates. Expert Opin. Drug Discov. 5 (2010) 609618.

[27] L. Di. Strategic Approaches to Optimizing Peptide ADME Properties. AAPS J. 17 (2015) 134-143.

[28] W. Li, J. Zhang, F.L. Tse. Strategies in quantitative LC-MS/MS analysis of unstable small molecules in biological matrices. Biomed. Chromatogr. 25 (2011) 258-277.

[29] L.T. Nguyen, J.K. Chau, N.A. Perry, L. de Boer, S.A. J. Zaat, H.J. Vogel. Serum stabilities of short tryptophan- and arginine-rich antimicrobial peptide analogs. PLoS One 5 (2010) 1-8.

[30] H. Hui, L. Farilla, P. Merkel, R. Perfetti. The short half-life of glucagon-like peptide-1 in plasma does not reflect its long lasting beneficial effects. Eur. J. Endocrinol. 146 (2002) 863-869.

[31] N. Tsomaia. Peptide therapeutics: Targeting the undruggable space. Eur. J. Med. Chem. 94 (2015) 459-470.

[32] Center for Drug Evaluation and Research. Guidance for Industry: Estimating the Maximum Safe Starting Dose in Initial Clinical Trials for Therapeutics in Adult Healthy Volunteers. US Dep. Heal. Hum. Serv. (2005) 1-27.

[33] D.F. McGinnity, J. Collington, R.P. Austin, R.J. Riley. Evaluation of Human Pharmacokinetics, Therapeutic Dose and Exposure Predictions Using Marketed Oral Drugs. Curr. Drug Metabol. 8 (2007) 463-479.

[34] E. Ezan. Pharmacokinetic studies of protein drugs: Past, present and future. Adv. Drug Deliv. Rev. 65 (2013) 1065-1073.

(C)2018 by the authors; licensee IAPC, Zagreb, Croatia. This article is an open-access article distributed under the terms and conditions of the Creative Commons Attribution license (http://creativecommons.org/licenses/by/3.0/) (cc)) EY 